\title{
Prediction of a two-dimensional crystalline structure of nitrogen atoms
}

\author{
V. Ongun Özçelik, ${ }^{1,2}$ O. Üzengi Aktürk, ${ }^{3}$ E. Durgun,,${ }^{1,2}$ and S. Ciraci ${ }^{4, *}$ \\ ${ }^{1}$ UNAM-National Nanotechnology Research Center, Bilkent University, 06800 Ankara, Turkey \\ ${ }^{2}$ Institute of Materials Science and Nanotechnology, Bilkent University, 06800 Ankara, Turkey \\ ${ }^{3}$ Department of Physics, Adnan Menderes University, 09100 Aydin, Turkey \\ ${ }^{4}$ Department of Physics, Bilkent University, 06800 Ankara, Turkey
}

(Received 14 February 2015; published 15 September 2015)

\begin{abstract}
Based on first-principles density functional calculations, we predict that nitrogen atoms can form a single-layer, buckled honeycomb structure called nitrogene, which is rigid and stable even above room temperature. This 2D crystalline phase of nitrogen, which corresponds to a local minimum in the Born-Oppenheimer surface, is a nonmagnetic insulator with saturated $\pi$ bonds. When grown on a substrate like $\mathrm{Al}(111)$ surface and graphene, nitrogene binds weakly to substrates and hence preserves its free-standing properties, but it can easily be pealed off. Zigzag and armchair nanoribbons of nitrogene have fundamental band gaps derived from reconstructed edge states. These band gaps are tunable with size and suitable for the emerging field of $2 \mathrm{D}$ electronics. Nitrogene forms not only bilayer, but also 3D graphitic multilayer structures. Single-layer nitrogene can nucleate and grow on the armchair edges of hexagonal boron nitride.
\end{abstract}

DOI: 10.1103/PhysRevB.92.125420

PACS number(s): 73.22.-f, 71.15.Mb, 77.55.-g, 81.05.Zx

\section{INTRODUCTION}

Finding the contender of graphene in the field of 2D electronics and in other potential applications has derived active research for graphenelike structures, which do not exist in nature. What followed graphene were other $2 \mathrm{D}$ materials, such as the 2D insulator boron-nitride (h-BN) [1-3], semimetallic silicene, germanene, and stanene, namely, graphene analogs of group IV elements [4-9], h-BN analogs of group IV-IV, III-V, and II-VI compounds [10], graphyne [11-14], transition-metal dichalcogenides [15-18], the auxetic piezoelectric 2D material silicatene with negative Poisson's ratio [19,20], etc. Much recently, the fabrication of field effect transistor using micrometer sized flakes consisting of two-three layers of black phosphorus [21] and theoretical analysis $[22,23]$ revealing the stability of its single-layer allotropes have made group $\mathrm{V}$ elements a subject of active research.

We performed a theoretical analysis to exploit the idea of whether other Group V elements like Sb, As, and N can form single-layer structures. Interestingly, the conclusion of our analysis has been positive and revealed that these three elements can also form 2D crystalline structures like silicene or phosphorene. Nitrogen, in particular, has a liquid phase at $77 \mathrm{~K}$; molecular/nonmolecular solid phases [24-29] and a $3 \mathrm{D}$ cubic gauche $(\mathrm{cg}-\mathrm{N})$ crystalline phase $[30,31]$ at extreme conditions, but normally it is gaseous at room temperature. Here we show that nitrogene can also form a crystalline phase with a $2 \mathrm{D}$ hexagonal lattice in a single-layer, buckled honeycomb structure, named nitrogene. Notably, while $\mathrm{N}_{2}$ molecule is triple bonded, nitrogene is constructed from threefold-coordinated and single-bonded $\mathrm{N}$ atoms similar to the $3 \mathrm{D}$ cg-N crystalline phase. We showed that nitrogene can remain stable above the room temperature and can form stable nanoribbons, bilayers, and a 3D graphitic structure named nitrogenite. However, unlike semimetallic graphene or silicene

*ciraci@fen.bilkent.edu.tr having perfect electron-hole symmetry, nitrogene is a wide band gap insulator. We believe that the present study is important not only because a contender of graphene is unveiled, but also for the prediction of a 2D single-layer crystal of nitrogen.

The organization of the paper is as follows. In Sec. II, we present the details of the methods used in our study. In Sec. III, we first describe the single-layer, buckled honeycomb structure of nitrogene obtained from structure optimized total energy calculations. Using ab initio phonon and finite temperature molecular dynamics calculations, we demonstrate that free nitrogene can remain stable above the room temperature. Furthermore, its stability is not affected by the formation of a single vacancy defect or adsorption of selected adatoms. In Sec. IV, we investigate the electronic structure of free nitrogene and its nanoribbons. Nitrogene bilayer and 3D layered structure and their electronic properties are also presented in the same section. In Sec. V, we present our simulation results related with the nucleation of nitrogene. We show that once grown on substrates like $\mathrm{Al}(111)$ or graphene, the interaction between nitrogene and the substrate becomes weak and hence the physical properties of free nitrogene are sustained.

\section{METHOD}

Our prediction of nitrogene is obtained from first-principles pseudopotential calculations based on the spin-polarized density functional theory (DFT) within the generalized gradient approximation including van der Waals (vdW) corrections at DFT-D2 level [32], which was found suitable for layered structures in earlier tests [33]. Similar calculations have been successful in predicting several 2D structures $[5,10,18,19]$, which were subsequently synthesized. We used projectoraugmented wave potentials [34] and the exchange-correlation was approximated with the Perdew-Burke-Ernzerhof (PBE) functional [35]. While optimizing the atomic positions using the conjugate gradient method (CG), the energy convergence value between two consecutive steps was chosen as $10^{-5} \mathrm{eV}$. A maximum force of $0.01 \mathrm{eV} / \AA$ was allowed on each atom. Calculations were carried out using the VASP software [36]. 
The Brillouin zone (BZ) corresponding to the primitive unit cell was sampled by a grid of $(21 \times 21 \times 1) \mathbf{k}$ points [37]. Since the band gaps are underestimated by standard DFT, we carried out calculations using the HSE06 hybrid functional [38], which is constructed by standard mixing $25 \%$ of the Fock exchange with $75 \%$ of the PBE exchange and $100 \%$ of the PBE correlation energy and also partially self-consistent quasiparticle $G W$ formalism $\left(G W_{0}\right)$ [39]. In addition to $a b$ initio phonon calculations [40], the stability of the structures in the local minimum was tested at high temperatures by ab initio, molecular dynamics (MD) calculations using two different approaches. In the first one, Nosé thermostat [41] is used and Newton's equations were integrated through Verlet algorithm with time step of $2 \mathrm{fs}$. In the second one, we scaled the velocities at each time step to keep the temperature constant.

\section{ATOMIC STRUCTURE AND STABILITY OF NITROGENE}

Among different possible geometries, the washboard structure of black phosphorene [22], which we adapted to nitrogene underwent an instability in the course of structure optimization and dissociated into $\mathrm{N}_{2}$ molecules. The planar honeycomb structure constructed from the $2 \mathrm{D}$ hexagonal lattice with two nitrogen atoms in the primitive cell is buckled in the course of structure optimizations; while the nitrogen atoms at three alternating corners of a planar hexagon are displaced downwards, the other three were raised upwards with a buckling distance of $\Delta=0.7 \AA$. The fully optimized nitrogene structure is described in Fig. 1. To avoid possible artifacts of single cell calculations, where reconstructions extending to few primitive cells can be hindered and thus the structure may fictitiously appear stable, we also optimized the structure using a $(4 \times 4)$ supercell providing higher degrees of freedom; this ended with the same structure obtained from the $(1 \times 1)$ singlecell optimization. The nitrogene structure has a nonmagnetic ground state. In the buckled honeycomb structure, three $s p^{n \alpha}$ orbitals of nitrogene atoms having bond angle $\alpha=99^{\circ}$ form covalent $\sigma$ bonds with the three nearest $\mathrm{N}$ atoms and provide the strength of nitrogene. The remaining two electrons on each nitrogen form $s p^{n \beta}$ orbitals with a relatively high $p$ contribution. They are involved in the $\pi$ - and $\pi^{*}$-like bonds with the nearest atoms and ensure the planar geometry constructed from two parallel planes of nitrogen atoms. The single bonding of nitrogene is illustrated by the isosurfaces of the total charge density in Fig. 1(a). Since the optimized structure of nitrogene has a cohesive energy of $E_{C}=3.67 \mathrm{eV} /$ atom, its formation energy relative to the strong $\mathrm{N}_{2}$ molecule is $-1.33 \mathrm{eV}$. Accordingly, nitrogene corresponds to a local minimum in the Born-Oppenheimer surface; it disintegrates to $\mathrm{N}_{2}$ molecules once an energy barrier is overcame. In this respect, the energy stored in the buckled honeycomb structure can be released suddenly like a $\mathrm{C}_{8} \mathrm{H}_{8}$ cubane molecule $[42,43]$ with inert gaseous nitrogen as the only product. The stored energy density is approximately equal to $130 \mathrm{~kJ} / \mathrm{kg}$, which is small when compared to common explosives.

At this point we compare the structural parameters and cohesive energy of nitrogene with similar single-layer structures of other group $\mathrm{V}$ elements, namely, $\mathrm{P}, \mathrm{As}$, and $\mathrm{Sb}$, all having buckled honeycomb structures. The nearest-neighbor distances of nitrogene, phosphorene [22], arsenene [44], and antimonene [45] are $1.49,2.27,2.50$, and $2.87 \AA$, the buckling values $0.70,1.24,1.39$, and $1.67 \AA$, and the cohesive energies per atom are 3.67, 5.19, 2.99, and $2.87 \mathrm{eV}$, respectively.

Even if the structure optimizations using CG favor stability of free-standing nitrogene, we perform further tests to assure that nitrogene is stable in the local minimum and can sustain applications above the room temperature. First, to verify the stability of nitrogene against small frequency acoustic vibrations and small transversal/longitudinal displacements in different directions, we calculate the frequencies of vibrational modes using a fine graded k-point sampling. All calculated frequencies are positive ensuring the stability of nitrogene. Otherwise, imaginary frequencies would imply structural instability for the corresponding modes. The vibrational bands along the symmetry directions of BZ are presented in Fig. 1(b). The optic branches are separated from acoustic branches, except at the $K$ point where one optical branch touches the acoustic one.

Even if all acoustic and ZA branches with positive frequencies ensure stability, the optimized structure by itself may correspond to a local shallow minimum and hence it may (a)

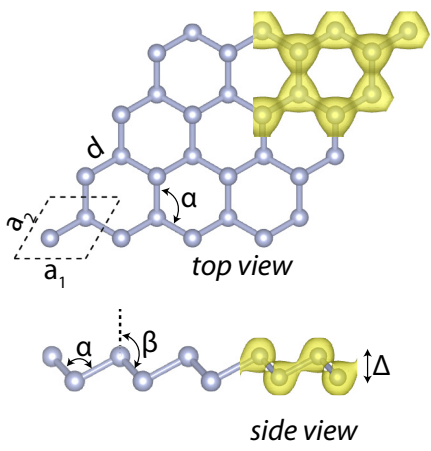

(b)

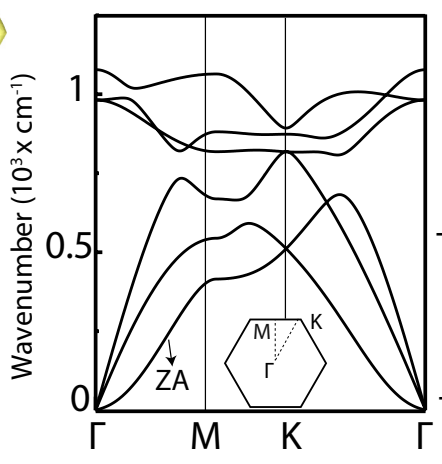

(c)

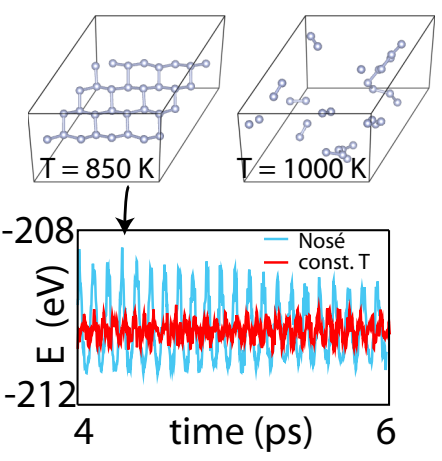

FIG. 1. (Color online) Structure and stability analysis of nitrogene: (a) 2D crystalline structure of nitrogene with optimized lattice constants $a_{1}=a_{2}=2.27 \AA$, the buckling $\Delta=0.7 \AA$, single-bonded N-N distance $d=1.49 \AA$, bond angles $\alpha=99^{\circ}$ and $\beta=118^{\circ}$. Bonding is depicted by an isosurface of the total charge density. (b) Vibrational bands. (c) Ab initio MD snapshots of atomic structure at temperatures 850 and $1000 \mathrm{~K}$. Energy vs time plots calculated using the Nosé thermostat and constant temperature value are shown with blue and red lines, respectively. 
turn to be unstable at a finite temperature. We clarified this critical situation by performing $a b$ initio $\mathrm{MD}$ calculations at 850 and $1000 \mathrm{~K}$. The stability is maintained at $850 \mathrm{~K}$ for $6 \mathrm{ps}$, but at $1000 \mathrm{~K}$ the crystalline structure was dissociated into $\mathrm{N}_{2}$ molecules as shown in Fig. 1(c). These MD calculations confirm at least the stability above the room temperature. Furthermore, we calculated the in-plane stiffness to be $C=$ $270 \mathrm{~N} / \mathrm{m}^{2}$, which indicates the strong covalency of N-N bonds in the buckled honeycomb structure. The calculated in-plane stiffness of nitrogene can be compared with those of graphene $\left(330 \mathrm{~N} / \mathrm{m}^{2}\right), \mathrm{h}-\mathrm{BN}\left(240 \mathrm{~N} / \mathrm{m}^{2}\right)$, silicene $\left(65 \mathrm{~N} / \mathrm{m}^{2}\right)$, and $\mathrm{MoS}_{2}$ $\left(138 \mathrm{~N} / \mathrm{m}^{2}\right)$, all having single-layer honeycomb structure. Apparently, nitrogene marks an in-plane stiffness value, which is the second highest among these nanostructures.

We next address the question whether the stability is endangered by the vacancy defect, or by the adsorption of single adatoms like $\mathrm{H}$ and $\mathrm{O}$. We consider a single vacancy periodically repeating in a $5 \times 5$ supercell of nitrogene, where the coupling with nearest defects is minute. Upon the optimization of atomic structure no rebonding took place between the two-folded nitrogen atoms surrounding the vacancy and hence the structure remained symmetric. The atoms surrounding the vacancy underwent a small relaxation, but a reconstruction observed in defect graphene [46-48] did not occur in nitrogene. The N-N bond lengths of the two-folded atoms reduce to $1.34 \AA$ from their equilibrium value of $1.49 \AA$ in the pristine structure. The vacancy formation is endothermic with a formation energy $E_{V}=5.59 \mathrm{eV}$. At the end, the defect structure remained stable by gaining a $3 \mu_{B}$ permanent magnetic moment per vacancy. The magnetic ground state of the defect structure complies with Lieb's theorem [49]. In addition, the buckled honeycomb structure of pristine nitrogene is preserved with a minute deformation at the close proximity of adsorbates such as $\mathrm{H}$ and $\mathrm{O}$ atoms, which are adsorbed with binding energies of 2.06 and $3.20 \mathrm{eV}$, respectively.

\section{ELECTRONIC STRUCTURE}

\section{A. Free nitrogene}

Having been assured of the stability of free nitrogene, we next investigate its electronic energy structure. Nitrogene is a nonmagnetic insulator with a DFT band gap of $E_{g}=3.96 \mathrm{eV}$. Because of honeycomb structure, the bands originating from $\pi$ - and $\pi^{*}$-like bonds cross each other and form cones around the $K$ point. However, in contrast to graphene and silicene, these $\pi$ and $\pi^{*}$ bands at the top of the valence band are filled, since the corresponding bonds are saturated. Calculations carried out by HSE show that the fundamental band gap is $E_{g, \mathrm{HSE}}=5.96 \mathrm{eV}$, hence DFT underestimates the band gap by $\sim 2 \mathrm{eV}$, whereas the many-body correction through the $G W_{0}$ method predicts a band gap of nitrogene of $7.26 \mathrm{eV}$, which apparently overestimates the HSE band gap by $1.3 \mathrm{eV}$ as seen in Fig. 2(a). The bands at the edges of conduction and valence bands are composed of mainly $2 p$-valence orbitals of nitrogen. The bands at the bottom of the valence band are dominated by $2 s$ orbitals. The charge density isosurfaces of $\pi$ and $\pi^{*}$ bands at the edge of the valence band clarify the role of the corresponding bonds in layer geometry. These bands are filled and separated from the empty bands by a large gap.

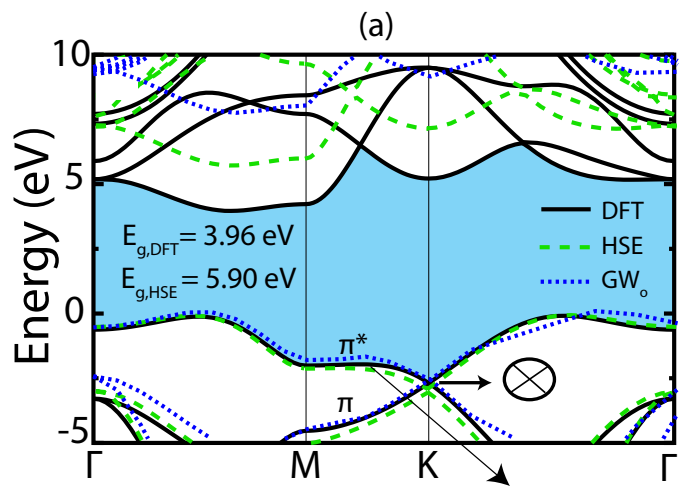

(b)

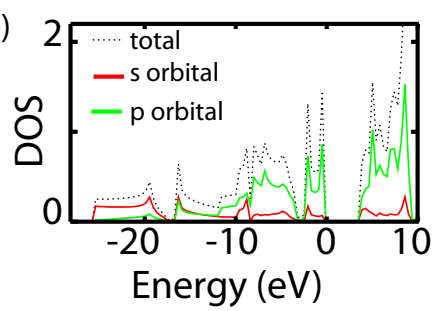

(c)

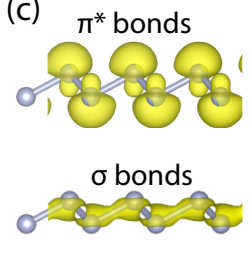

FIG. 2. (Color online) Free nitrogene: (a) Electronic band structure, (b) Total and $s$ - and $p$-orbital projected densities of states, and (c) The charge density isosurfaces of states associated with $\pi^{*}$ and $\sigma$ bonds. The bands calculated by HSE and $\mathrm{GW}_{o}$ are shown with green dashed and blue dotted lines. The crossing at the $K$ point is highlighted. The zero of energy is set to the top of the valence band.

Single vacancy formation in nitrogene gives rise to three filled spin-down states and three empty spin-up states as defect states. These states are localized in the band gap and have a gap $\sim 0.4 \mathrm{eV}$ between them, and $3 \mu_{B}$ permanent magnetic moment. The energy position of these localized gap states and the resulting high magnetic moments can be monitored by the decoration of vacancy in diverse patterns.

\section{B. Nitrogene nanoribbons}

Since finite size ribbons are used in various applications, their stability and electronic properties become critical for the characterization of nitrogene. Here, we consider bare nitrogene nanoribbons having armchair and zigzag edge geometries, which are specified by the number of nitrogen atoms, $n$ in their primitive unit cell. In Fig. 3(a), we show the primitive unit cell of the armchair nanoribbon with $n=22$ and the corresponding band structure with $E_{g}=2.16 \mathrm{eV}$ direct band gap. The variation of $E_{g}$ with the width of the ribbon or $n$ shows a family behavior, especially for small $n$. The band gap $E_{g}$ is small for low values of $n$, but it gradually increases with decreasing coupling between edges for large $n$, and eventually saturates at $E_{g} \sim 2.2 \mathrm{eV}$ for $n>22$. Two bands at the edge of valence and conduction bands ( $\mathrm{V}$ and $\mathrm{C}$ ) are derived from the edge states as demonstrated by the band decomposed charge density isosurfaces. The direct band gap of the armchair nanoribbon with $n=22$ increases $\sim 1.3 \mathrm{eV}$ upon HSE correction.

The edge atoms of the zigzag nanoribbon undergo a reconstruction. The structure optimization using a primitive unit cell with $n$ atoms mandates a fictitious, metallic structure. However, upon a Peierls type structural transformation, the 
(a)
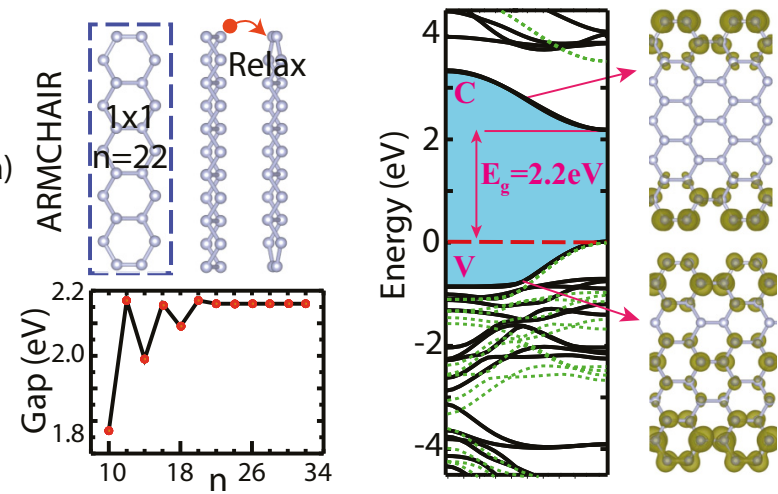

(b)

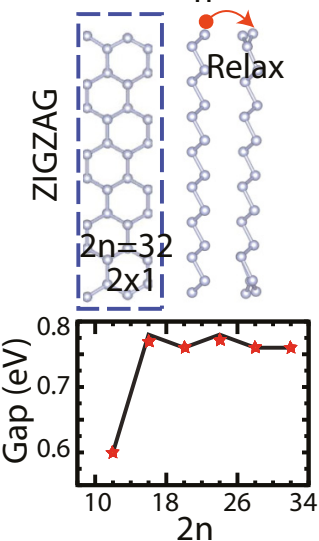

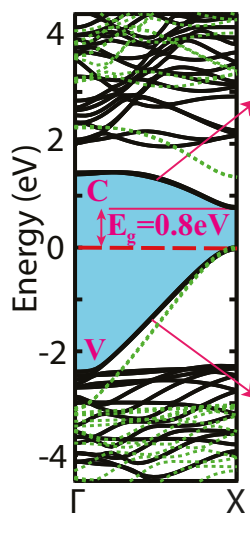

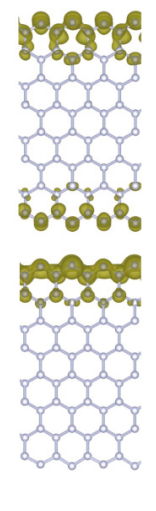

FIG. 3. (Color online) Bare nanoribbons of nitrogene: (a) Atomic configuration and the energy band structure of the armchair nitrogene nanoribbon specified by the number of nitrogen atoms in the primitive unit cell, $n$. Variation of band gap with $n$ and charge density isosurfaces of specific band states at the edges of conduction (C) and valence $(\mathrm{V})$ bands are shown. Energy bands calculated by HSE are shown by dashed lines. (b) Same for the zigzag nanoribbon, where calculations are performed for the $2 \times 1$ unit cell.

(a)

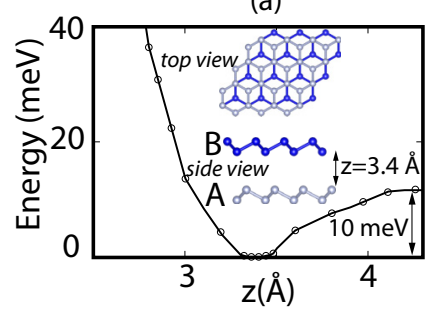

(c)

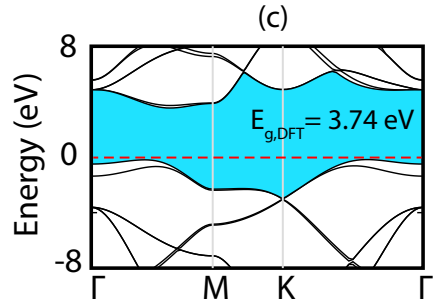

(b)

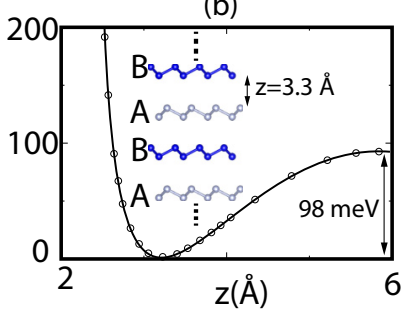

(d)

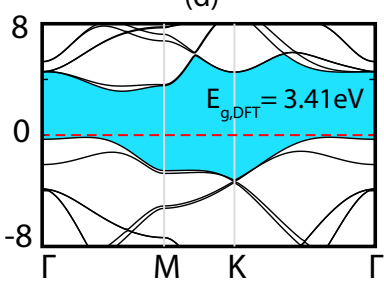

FIG. 4. (Color online) Bilayer and 3D layered structures: (a) Minimum energy stacking geometry of bilayer and the variation of the binding energy of layers with the distance $z$ between them. The binding energies of layers $E_{b}$ are given relative to $z \rightarrow \infty$. (b) Same for $3 \mathrm{D}$ nitrogenite. The electronic band structures of bilayer (c) and nitrogenite $(d)$.

metallic state changes to semiconductor. In Fig. 3(b), we present the reconstructed structure of the zigzag nanoribbon with $2 n=32$, which is optimized in the $2 \times 1$ unit cell. The corresponding band gap calculated by DFT is $E_{g} \sim 0.76 \mathrm{eV}$. This value increases by $\sim 0.5 \mathrm{eV}$ upon HSE correction. However, the band gap varies with $2 n$; it is small for low values of $2 n$, but increases and saturates to $E_{g}=0.75 \mathrm{eV}$ for $2 n>32$ as presented in Fig. 3(b). Accordingly, the band gap values of zigzag nanoribbons are found to be convenient for 2D electronic applications. The charge density isosurfaces of $\mathrm{V}$ and $\mathrm{C}$ bands clearly show that they are associated with

(a)

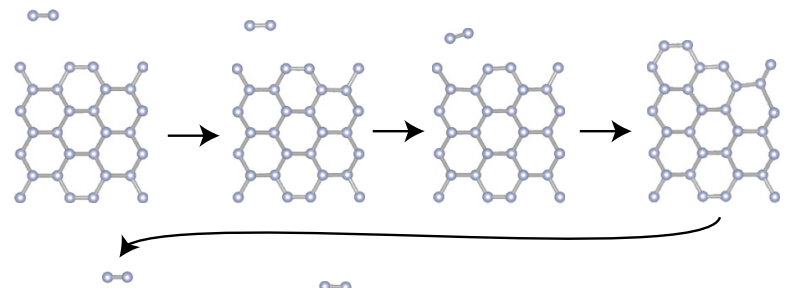

(b)

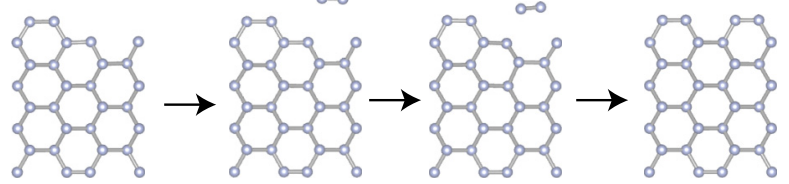

(c)
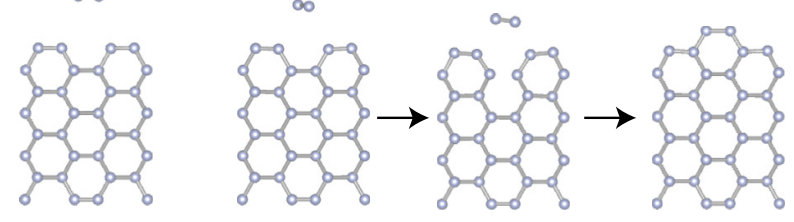

(d)

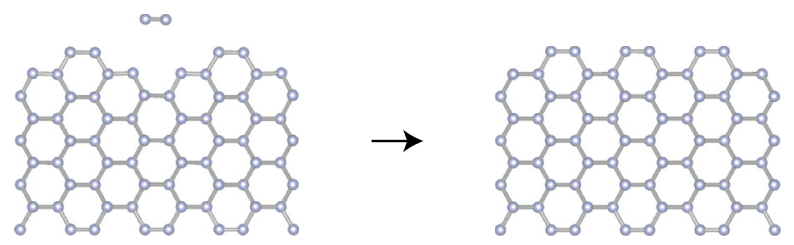

(e)

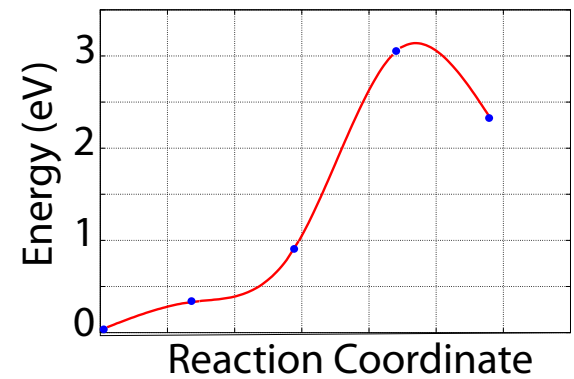

FIG. 5. (Color online) Snapshots of atomic configurations corresponding to the nucleation and initial stages of growth of nitrogene from the armchair edge of a nitrogene seed (or small flake) calculated by the nudge elastic band (NEB) method. Each snapshot corresponds to an intermediate step. (a) and (b) Initial $\mathrm{N}_{2}$ molecules approach the seed and construct nitrogen hexagons. (c) and (d) The second row hexagons are forming. (e) Energy barrier calculated by NEB method for the formation of nitrogene from $\mathrm{N}_{2}$ molecule. 
edge states. Both armchair and zigzag nanoribbons have a nonmagnetic ground state; as an example, the nonmagnetic zigzag nanoribbon with $2 n=32$ is more favorable than the magnetic state by $394 \mathrm{meV}$. In contrast to $\pi$ and $\pi^{*}$ bonds of nitrogene, the bonds of two folded atoms at the edge of nanoribbons are chemically active and can form strong bonds with foreign atoms. Upon the saturation of nitrogene at the edges by hydrogen atoms, the bands associated with edge states are discarded and the DFT band gap increases to $\sim 4 \mathrm{eV}$ for both armchair and zigzag nanoribbons.

\section{Bilayer and multilayer nitrogene}

Our analysis shows that not only single-layer nitrogene, but also bilayer and its 3D graphitic phase constructed from the stacking of single nitrogene layers, namely nitrogenite are stable. The relative lateral displacement of adjacent nitrogene layers by $\mathbf{r}=\left(\mathbf{a}_{1}+\mathbf{a}_{2}\right) / 3$ leads to the minimum energy, $\mathrm{AB}$ stacking for the bilayer. Similarly, the ABAB ... stacking for 3D nitrogenite has the minimum energy. Accordingly, the three nitrogen atoms at the alternating corners of a hexagon face the similar nitrogen atoms of the adjacent layers, while the remaining three nitrogen atoms face the centers of hexagons. This way, the N-N bonds in each nitrogene layer become staggered relative to the ones in the adjacent layers. The stacking geometry of layers described in Figs. 4(a) and $4(\mathrm{~b})$ is reminiscent of graphene stacking in graphite. In the same figure, the variation of total energy relative to the spacing $z$ between two layers is shown; the minimum energy occurs for $z=3.38 \AA$, and the binding energy of layers is weak and is only $E_{b}=10 \mathrm{meV} /$ cell. However, owing to increased interlayer interactions from both sides, nitrogenite has relatively higher interlayer binding energy (or exfoliation energy) $E_{b}=98 \mathrm{meV} /$ cell and a relatively smaller interlayer distance of $z=3.26 \AA$ relative to the bilayer. The weak interlayer interaction is predominantly $\mathrm{vdW}$ attraction. Consequently, as shown in Figs. 4(c) and 4(d), the electronic band structure of bilayer and nitrogenite are not affected significantly by the weak coupling between adjacent nitrogene layers. The DFT band gaps are slightly modified in the multilayer structures of nitrogene.

\section{NUCLEATION OF NITROGENE}

While the triple bonded diatomic nitrogen $\mathrm{N}_{2}$ has strong binding energy and is in a gaseous state at room temperature, its crystalline and molecular solid phases have been an active subject of study. Earlier, theoretical studies based on DFT total energy calculations using psedopotentials with a plane-wave basis set predicted metallic, polymeric, and diatomic phases of nitrogen [27]. In particular, a crystalline form with a cubic gauche structure was predicted [27]. Later, Raman, infrared and X-ray measurements provided evidence for new phases of nitrogen at high pressure and temperature [29]. Some of these phases were quenched to room temperature. At low temperatures and pressures, nitrogen forms an insulating molecular crystal in which $\mathrm{N}_{2}$ molecules are held together by weak vdW attractive interactions [50]. As the pressure is raised, $\mathrm{N}_{2}$ molecules dissociate to form mono-atomic metallic solids. This nonmolecular phase is a semiconductor at room temperature at 140 GPA pressure. Notably, this phase remains stable and becomes a semiconductor at low temperatures below $100 \mathrm{~K}$ and ambient pressure for practical usage [30]. Additionally, a crystalline form of nitrogen, where the atoms are connected with single covalent bonds, is synthesized recently at extreme conditions [31]. This is the theoretically predicted cubic gauche structure, which has $\sim 1 \mathrm{eV} /$ atom energy above the energy of the diatomic $\alpha-\mathrm{N}_{2}(\mathrm{~Pa} \overline{3})$ phase [27].

The present study predicts that a $2 \mathrm{D}$, single-layer crystal of single-bonded $\mathrm{N}$ atoms with a buckled honeycomb structure, namely nitrogene, can form as a local minimum and remain stable above room temperature. Here, based on $a b$ initio simulations we show two atomic mechanisms of how nitrogene can nucleate. Both mechanisms provide evidence that a nitrogene crystal can be synthesized.

The first mechanism starts from a seed (or a small flake) of nitrogene, which has an armchair edge. As shown in Figs. 5(a)-5(d), nitrogene can nucleate and grow by the intake of $\mathrm{N}_{2}$. Using the nudge elastic band (NEB) method [51], the corresponding energy barrier is calculated to be $\sim 3 \mathrm{eV}$ in Fig. 5(e). In the second mechanism, the nucleation of nitrogene can be realized also at the armchair edges of single-layer, hexagonal BN (h-BN) seeds or small flakes. Snapshots of atomic configurations at different stages of the nucleation and growth, which are obtained through the $a b$ initio simulations are described in Fig. 6. The grown nitrogene has the Stone-Wales defects consisting of adjacent five-fold and seven-fold rings. In the advanced stages, large rings even chains of $\mathrm{N}$ atoms can form. Interestingly, similar situations have occurred also in the growth of graphene on h-BN [52]. These defects are healed in the course of prolonged

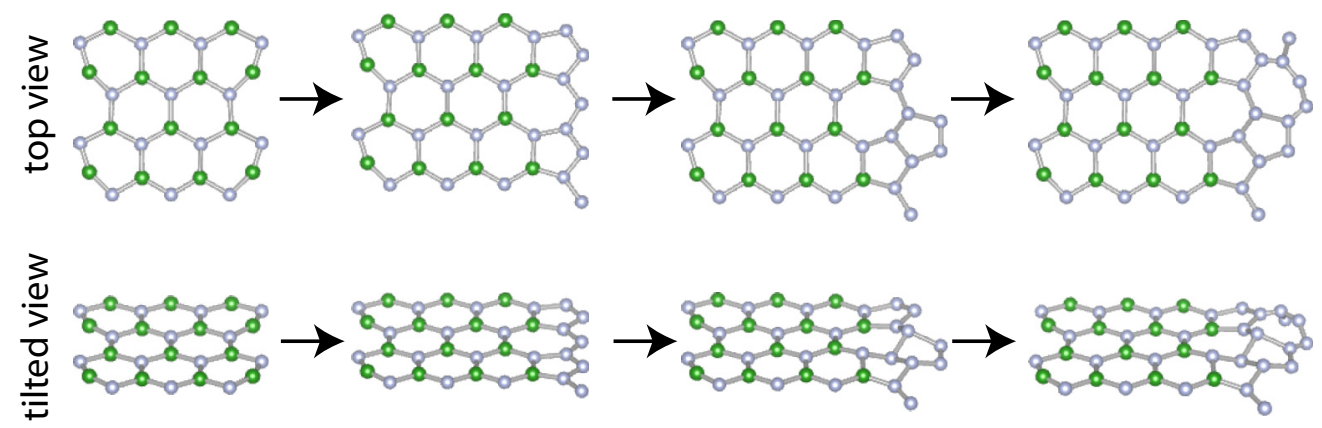

FIG. 6. (Color online) Top and tilted views of snapshots of atomic configurations obtained from the simulation of the nucleation of nitrogene through the intake of $\mathrm{N}_{2}$ at the armchair edge of a h-BN seed. 
cycles of growth. Since small h-BN flakes can easily be transferred on a suitable substrate, the second mechanism appears rather promising. Other scenarios including aggregation within a layered template structure such as h-BN, where the activation barrier may be lowered catalytically, are also possible.

\section{A. Nitrogene on substrates}

Here, we address the questions whether nitrogene remains stable and maintains its free standing properties once it is grown on a suitable substrate. We first examine the interaction between a reactive metal substrate and nitrogene. To this end, we consider an $\mathrm{Al}(111)$ surface despite other substrate surfaces that may have lattice constants close to that of nitrogene, since $\mathrm{Al}(111)$ is rather reactive. To compensate the $7 \%$ lattice mismatch, we compress $\mathrm{Al}(111)$ uniformly.

Atomic configuration of nitrogene on an $\mathrm{Al}(111)$ substrate, which is mimicked by a slab consisting of four $\mathrm{Al}(111)$ atomic planes as described in Fig. 7(a). The binding energy of nitrogene to the $\mathrm{Al}(111)$ substrate is $190 \mathrm{meV} /$ atom and consequently the minimum distance between nitrogene and $\mathrm{Al}$ surface atoms is large and is $3.1 \AA$. This situation suggests that nitrogene interacts weakly even with a reactive metal surface through a vdW attraction. The calculated total density of states of the nitrogene $+\mathrm{Al}(111)$ system, the local density of states at the nitrogene overlayer, and at the $\mathrm{Al}(111)$ substrate are shown in Fig. 7(b). The local density of states at the nitrogene overlayer is almost identical to the total density of states of free nitrogene presented in Fig. 2(b) and has a fundamental band gap of $3.9 \mathrm{eV}$. This is strong evidence that the electronic structure of free-standing nitrogene discussed in Sec. IV is sustained after the growth of a single nitrogene layer on a reactive metal substrate. On the other hand, the local density of states on the $\mathrm{Al}(111)$ slab indicates that it remains metal with a high density of states at the Fermi level. Moreover, the $\mathrm{Al}(111)$ slab comprising a finite number of $\mathrm{Al}(111)$ planes has a density of states that displays a stepwise variation with energy as a manifestation of the quantum size effect [53]. In the calculated band structure of nitrogene grown on an $\mathrm{Al}(111)$ surface in Fig. 7(c) one distinguishes nitrogene bands with a 3.9-eV band gap from the metallic $\mathrm{Al}(111)$ slab bands. The bands belonging to the nitrogene overlayer and $\mathrm{Al}(111)$ slab are separated in the momentum space. Therefore the conduction electrons of the $\mathrm{Al}(111)$ slab encounter a barrier to join the conduction band of nitrogene. In summary, the local density of states and the electronic band structure corroborate the weak interaction between the nitrogene overlayer and the Al(111) slab, and indicate that the properties of nitrogene grown on the surface of a reactive metal like $\mathrm{Al}$ do not alter from those of free nitrogene. Notably, the equilibrium binding energy of fully relaxed nitrogene on a graphene substrate is even weaker and is only $39 \mathrm{meV} /$ atom.

\section{CONCLUSIONS}

In conclusion, our theoretical study predicts that nitrogen atoms can form a 2D single-layer crystal with a hexagonal lattice and buckled honeycomb structure. This structure named as nitrogene shares the same structure common to the stable (a)

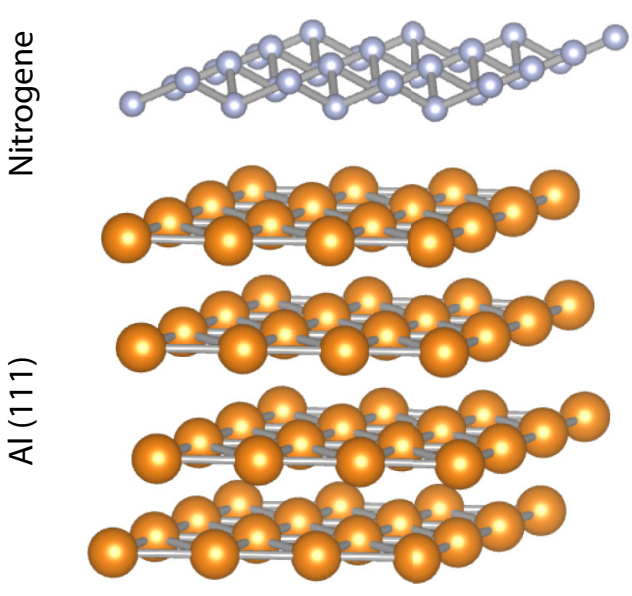

(b)

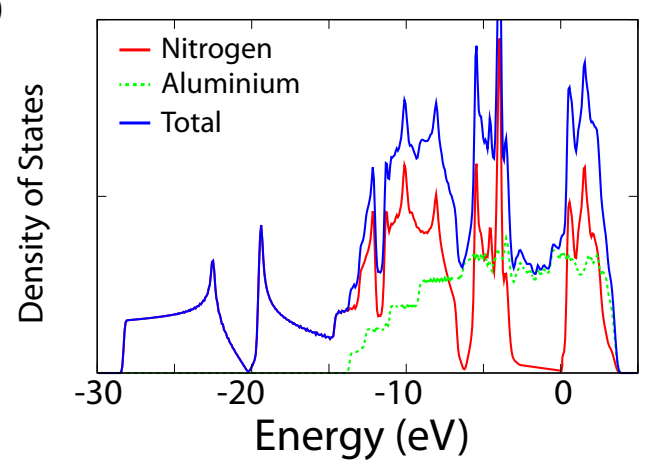

(c)

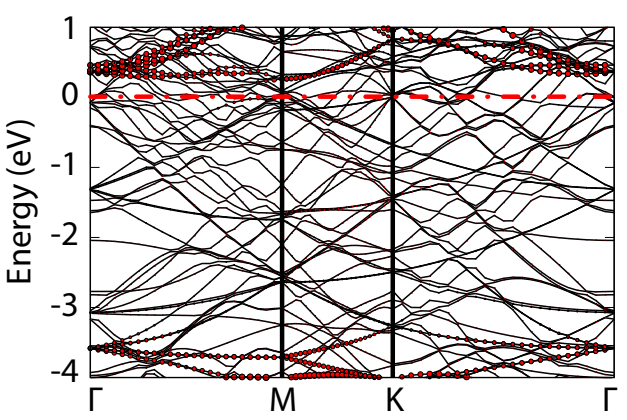

FIG. 7. (Color online) Nitrogene on $\mathrm{Al}(111)$ substrate: (a) Atomic configuration of nitrogene overlayer on the $\mathrm{Al}(111)$ substrate (or slab) consisting of four $\mathrm{Al}(111)$ planes. Small (light blue) and large (yellow) balls denote $\mathrm{N}$ and $\mathrm{Al}$ atoms, respectively. (b) Total and local density of states projected on $\mathrm{N}$ and $\mathrm{Al}$ atoms. Nitrogene layer on $\mathrm{Al}(111)$ preserves its fundamental gap, while the metallic density of states of Al slab shows a stepwise variation with energy. (c) Electronic band structure of nitrogene $+\mathrm{Al}(111)$ system. The states of nitrogene layer are indicated by dotted (red) lines.

single-layer structures of group V elements, i.e., phosphorene, arsenene, and antimonene. Free nitrogene is stable in ambient conditions and remains intact above the room temperature. While pristine nitrogene is a wide band gap insulator, its nanoribbons with zigzag and armchair edge geometries have band gaps in the range of $0.6 \mathrm{eV}<E_{g}<2.2 \mathrm{eV}$, which can be engineered by varying their widths. Additionally, nitrogene can form a stable bilayer, as well as a 3D layered 
structure named nitrogenite. The results of our $a b$ initio simulations and NEB [51] calculations suggest that nitrogene can nucleate at the edges of nitrogene and h-BN seeds. When grown on substrates, nitrogene interacts weakly even with reactive metal substrates and maintains the properties of free nitrogene. Briefly, the present study predicts a stable 2D crystal of nitrogen atoms, which forms also 3D layered solid, bilayer, and 1D ribbon structures. These structures can function either in 2D electronics or in the coating of specific surfaces.

\section{ACKNOWLEDGMENTS}

The computational resources are provided by TUBITAK ULAKBIM, High Performance and Grid Computing Center (TR-Grid e-Infrastructure). VOÖ and SC acknowledge financial support from the Academy of Sciences of Turkey (TUBA). This work is partially supported by TUBITAK under the Project No. 113T050. ED acknowledges support from Bilim Akademisi - The Science Academy, Turkey under the BAGEP program.
[1] K. S. Novoselov, D. Jiang, F. Schedin, T. J. Booth, V. V. Khotkevich, S. V. Morozov, and A. K. Geim, Proc. Natl. Acad. Sci. USA 102, 10451 (2005).

[2] D. Pacilé, J. C. Meyer, Ç. Ö. Girit, and A. Zettl, Appl. Phys. Lett. 92, 133107 (2008).

[3] M. Topsakal, E. Aktürk, and S. Ciraci, Phys. Rev. B 79, 115442 (2009).

[4] E. Durgun, S. Tongay, and S. Ciraci, Phys. Rev. B 72, 075420 (2005).

[5] S. Cahangirov, M. Topsakal, E. Aktürk, H. Sahin, and S. Ciraci, Phys. Rev. Lett. 102, 236804 (2009).

[6] P. Vogt, P. DePadova, C. Quaresima, J. Avila, E. Frantzeskakis, M. C. Asensio, A. Resta, B. Ealet, and G. LeLay, Phys. Rev. Lett. 108, 155501 (2012).

[7] V. O. Özçelik, E. Durgun, and S. Ciraci, J. Phys. Chem. Lett. 5, 2694 (2014).

[8] Y. Xu, B. Yan, H. J. Zhang, J. Wang, G. Xu, P. Tang, W. Duan, and S. C. Zhang, Phys. Rev. Lett. 111, 136804 (2013).

[9] L. Tao, E. Cinquanta, D. Chiappe, C. Grazianetti, M. Fanciulli, M. Dubey, A. Molle, and D. Akinwande, Nat. Nanotech. 10, 227 (2015)

[10] H. Sahin, S. Cahangirov, M. Topsakal, E. Bekaroglu, E. Akturk, R. T. Senger, and S. Ciraci, Phys. Rev. B 80, 155453 (2009).

[11] S. Tongay, S. Dag, E. Durgun, R. T. Senger, and S. Ciraci, J. Phys. Condens. Matter 17, 3823 (2005).

[12] D. Malko, C. Neiss, F. Viñes, and A. Görling, Phys. Rev. Lett. 108, 086804 (2012).

[13] V. O. Özçelik and S. Ciraci, J. Phys. Chem. C 117, 2175 (2013).

[14] N. K. Perkgoz and C. Sevik, Nanotechnol. 25, 185701 (2014).

[15] P. Joensen, R. F. Frindt, and S. R. Morrison, Mater. Res. Bull. 21, 457 (1986).

[16] J. N. Coleman, M. Lotya, A. O’Neill, S. D. Bergin, P. J. King, U. Khan, and K. Young, Science 331, 568 (2011).

[17] C. Ataca and S. Ciraci, J. Phys. Chem. C 115, 13303 (2011).

[18] C. Ataca, H. Sahin, and S. Ciraci, J. Phys. Chem. C 116, 8983 (2012).

[19] V. O. Özçelik, S. Cahangirov, and S. Ciraci, Phys. Rev. Lett. 112, 246803 (2014).

[20] X. Xu, J. Zhuang, Y. Du, H. Feng, N. Zhang, C. Liu, T. Lei, J. Wang, M. Spencer, T. Morishita, X. Wang, and S. X. Dou, Sci. Rep. 4, 7543 (2014).

[21] L. Li, Y. Yu, G. J. Ye, Q. Ge, X. Ou, H. Wu, D. Feng, X. H. Chen, and Y. Zhang, Nat. Nanotechnol. 9, 372 (2014).

[22] Z. Zhu and D. Tomanek, Phys. Rev. Lett. 112, 176802 (2014).
[23] T. Low, A. S. Rodin, A. Carvalho, Y. Jiang, H. Wang, F. Xia, and A. H. Castro Neto, Phys. Rev. B 90, 075434 (2014).

[24] R. L. Mills and A. F. Schuch, Phys. Rev. Lett. 23, 1154 (1969).

[25] W. J. Nellis, N. C. Holmes, A. C. Mitchell, and M. van Thiel, Phys. Rev. Lett. 53, 1661 (1984).

[26] A. K. McMahan and R. LeSar, Phys. Rev. Lett. 54, 1929 (1985).

[27] C. Mailhiot, L. H. Yang, and A. K. McMahan, Phys. Rev. B 46, 14419 (1992).

[28] L. Mitas and R. M. Martin, Phys. Rev. Lett. 72, 2438 (1994).

[29] E. Gregoryanz, A. F. Goncharov, R. J. Hemley, H.-k. Mao, M. Somayazulu, and G. Shen, Phys. Rev. B 66, 224108 (2002).

[30] M. I. Eremets, R. J. Hemley, H. Mao, and E. Gregoryanz, Nature (London) 411, 170 (2001).

[31] M. I. Eremets, A. G. Gavriliuk, I. A. Trojan, D. A. Dzivenko, and R. Boehler, Nat. Mater. 3, 558 (2004).

[32] S. Grimme, J. Comput. Chem. 27, 1787 (2006).

[33] C. Ataca, M. Topsakal, E. Akturk, and S. Ciraci, J. Phys. Chem. C 115, 16354 (2011).

[34] P. E. Blöchl, Phys. Rev. B 50, 17953 (1994).

[35] J. P. Perdew, K. Burke, and M. Ernzerhof, Phys. Rev. Lett. 77, 3865 (1996).

[36] G. Kresse and J. Furthmüller, Phys. Rev. B 54, 11169 (1996).

[37] H. J. Monkhorst and J. D. Pack, Phys. Rev. B 13, 5188 (1976).

[38] J. Paier, M. Marsman, K. Hummer, G. Kresse, I. C. Gerber, and J. G. Ángyán, J. Chem. Phys. 124, 154709 (2006).

[39] M. Shishkin and G. Kresse, Phys. Rev. B 75, 235102 (2007).

[40] D. Alfe, Comput. Phys. Commun. 180, 2622 (2009).

[41] S. Nosé, J. Chem. Phys. 81, 511 (1984).

[42] P. E. Eaton, Angew Chem. 31, 1421 (1992).

[43] T. Yildirim, C. Kilinc, S. Ciraci, P. M. Gehring, D. A. Neumann, P. E. Eaton, and T. Emrick, Chem. Phys. Lett. 309, 234 (1999); C. Kilinc, T. Yildirim, H. Mehrez, and S. Ciraci, J. Phys. Chem. A 104, 2724 (2000).

[44] C. Kamal and M. Ezawa, Phys. Rev. B 91, 085423 (2015).

[45] O. U. Akturk, V. O. Ozcelik, and S. Ciraci, Phys. Rev. B 91, 235446 (2015).

[46] O. V. Yazyev and L. Helm, Phys. Rev. B 75, 125408 (2007).

[47] A. W. Robertson, B. Montanari, K. He, C. S. Allen, Y. A. Wu, N. M. Harrison, A. I. Kirkland, and J. H. Warner, ACS Nano 7, 4495 (2013).

[48] V. O. Özçelik, H. H. Gurel, and S. Ciraci, Phys. Rev. B 88, 045440 (2013).

[49] E. H. Lieb, Phys. Rev. Lett. 62, 1201 (1989). 
[50] Y. A. Freiman, in Physics of Cryocrystals, edited by V. G. Manzhelli and Y. A. Freiman (American Institute of Physics, Woodbury, New York, 1997), Vol. 538.

[51] G. Henkelman, B. P. Uberuaga, and H. Jónsson, J. Chem. Phys. 113, 9901 (2000).
[52] V. O. Özçelik and S. Ciraci, Phys. Rev. B 86, 155421 (2012).

[53] S. Ciraci and I. P. Batra, Phys. Rev. B 33, 4294 (1986). In a 2D free electron gas, the density of states is constant. A four-layer slab can be considered as 2D; each band dipping in the Fermi level gives rise to a constant jump proportional to $m / \pi \hbar^{2}$. 